\title{
Unilateral Multicanalicular Vestibular Lithiasis of Right Horizontal and Posterior Semicircular Canals: An Unusual Case
}

\author{
Ajay Kumar Vats ${ }^{1 \oplus \odot}$ \\ ${ }^{1}$ Medicine and Neurology Department, Chaudhary Hospital \& \\ Medical Research Centre Private Limited, Udaipur, Rajasthan, India
}

Address for correspondence Ajay Kumar Vats, MBBS, MD (Medicine), DM (Neurology), FRCP, Chaudhary Hospital \& Medical Research Centre Private Limited, 472-473, Sector 4, Hiran Magri, Udaipur, Rajasthan, 313002, India (e-mail: vatsneuro@gmail.com).

\begin{abstract}
Keywords

- vestibular lithiasis

- multicanalicular

- otoconial debris

- BPPV

- Dix-Hallpike test

- supine head roll test

Vestibular lithiasis (canalolithiasis as well as cupulolithiasis) commonly exists in monocanalicular forms involving one of the three semicircular canals, frequent posterior, less frequent horizontal, and very rarely anterior. It is treated with canal clearing maneuvers intended to reposition the otoconia from the semicircular canal (where they have inappropriately entered) through the utricular exit in the nonampullary arm of the semicircular canal to the utricle (where they normally remain as a part of utricular gelatinous matrix). The cases of multicanalicular vestibular lithiasis with the involvement of more than one semicircular canal require meticulous identification of the involved canals and multiple different canal-clearing maneuvers for effective treatment. A 70-year-old male patient with no significant history of previous medical or otologic illnesses or head trauma presented with a 1-day history of vertigo with positional aggravation. A one-time performed diagnostic supine head roll test elicited three different patterns of positional nystagmus, each with an accurate localizing and lateralizing value. Diagnosis of unilateral multicanalicular vestibular lithiasis of right horizontal and posterior semicircular canals was entertained based on the pattern of the elicited positional nystagmi on the supine roll test. The upbeating torsional nystagmus that localizes the involvement to the posterior semicircular canal was paradoxically elicited by supine head roll test and not by the Dix-Hallpike test. As horizontal semicircular canalolithiasis causes severe symptoms, its treatment preceded that of concurrent posterior semicircular canalolithiasis. The patient was successfully treated with multiple sessions of canalith repositioning maneuvers (CRMs) spread over 24 hours. It is important to perform both positional tests, namely Dix-Hallpike maneuver, and supine head roll test, in cases suspected to have multicanalicular vestibular lithiasis. The positionings may need to be repeated several times to unveil multiple nystagmi, each with different localizing and lateralizing values. Identifying treatment priorities with CRM for the individual semicircular canals is crucial, and the canal that is liable to cause severe symptoms needs early clearance of the otoconial debris. If a CRM fails to clear a semicircular canal, an alternative maneuver may need to be executed. Clinicians involved in the care of cases with multicanalicular vestibular lithiasis should be well versed with all possible backup maneuvers for clearing each of the three semicircular canals.
\end{abstract}

Published online

September 29, 2021
DOI https://doi.org/ 10.1055/s-0041-1735989 ISSN 2581-9607
(C) 2021. Indian Society of Otology.

This is an open access article published by Thieme under the terms of the Creative Commons Attribution-NonDerivative-NonCommercial-License, permitting copying and reproduction so long as the original work is given appropriate credit. Contents may not be used for commercial purposes, or adapted, remixed, transformed or built upon. (https://creativecommons.org/licenses/by-nc-nd/4.0/).

Thieme Medical and Scientific Publishers Pvt. Ltd. A-12, 2nd Floor,

Sector 2, Noida-201301 UP, India 


\section{Introduction}

Vestibular lithiasis exists either as free-floating otoconia within the semicircular canal (SCC) (canalolithiasis) or as otoconial particles getting adherent to the cupula (cupulolithiasis), thus making it heavy and gravity sensitive. Cupulolithiasis also exists in two forms, with the otoconial particles getting attached either to the canal side (Cup-C) or to the utricular side (Cup-U) of the cupula. - Table 1 shows that 0 to $21.25 \%$ of all patients diagnosed with benign paroxysmal positional vertigo (BPPV) at any specialty clinic have multicanalicular BPPV. ${ }^{1-11}$ In multicanalicular BPPV, either there is the involvement of the same SCC on both sides or simultaneous involvement of different SCCs on the same or both sides. Multicanalicular BPPV frequently occurs with underlying comorbid conditions like head trauma, labyrinthitis, Meniere's disease, and otitis media. ${ }^{12-16}$

Multicanalicular BPPV has been subclassified as under: ${ }^{13}$

1. Single-canal bilateral involving the same SCC in either of the labyrinths.

2. Multicanal unilateral involving at least two different SCCs (posterior, lateral, or anterior) in one of the labyrinths.

3. Multicanal bilateral involving two or more different SCCs in both labyrinths.

Multicanalicular BPPV is unilateral in two-thirds of the cases, bilateral in the other one-third of the cases, and roughly 80 to $90 \%$ of these patients have simultaneous involvement of posterior (PSC) and horizontal semicircular canal (HSC) of the same or opposite side. ${ }^{13,17}$ A mixed geotropic-horizontal and upbeating nystagmus with a disproportionately large geotropic-torsional component toward the lowermost ear either in the Dix-Hallpike or supine roll test is indicative multicanalicular BPPV, affected with concurrent posterior and horizontal semicircular canalolithiasis. ${ }^{18}$ However, in a study of 20 patients diagnosed with monocanalicular horizontal semicircular canalolithiasis, the use of three-dimensional video-oculography demonstrated a vertical component in 15
(75\%) and a torsional component in 19 (95\%) patients. ${ }^{19}$ The variant single-canal bilateral BPPV involving anterior SCCs is a difficult-to-diagnose condition because the lateralizing torsional component of positional downbeat nystagmus is often inconspicuous or absent. Bilateral HSC-BPPV, with its various possible permutation and combinations of canalolithiasis and cupulolithiasis on either side, is also potentially difficult to diagnose. Likewise, in the PSC-BPPV, although the Dix-Hallpike test might be positive bilaterally, the disease is likely to be bilateral only if there is a torsional component clockwise on one side and counterclockwise on the other. ${ }^{20}$

The positional maneuvers, namely Dix-Hallpike maneuver for the vertical canals (PSC and anterior SCCs) and supine roll test for the HSCs, need to be meticulously performed on both sides in all cases of BPPV (idiopathic as well posttraumatic). The Dix-Hallpike maneuver is considered specific for the diagnosis of the PSC-BPPV, but in as many as $80 \%$ of cases, it may elicit the positional nystagmus of HSC-BPPV as well. ${ }^{21}$ The elicited positional nystagmus localizes the involved SCC as per the Ewald's first law, which states that the vestibular nystagmus is always directed parallel to the plane of the stimulated canal. ${ }^{22}$ The variations in the geometry of SCC among individuals might be responsible for the elicitation of positional nystagmus of the HSC during the Dix-Hallpike test. Therefore, it is imperative to carry out the supine roll test with 30 degrees of cervical flexion in all cases of suspected BPPV, where the Dix-Hallpike maneuver to either of the sides has failed to elicit any positional nystagmus. ${ }^{23}$ Video recording of diagnostic positional test and replaying it on the bigger screen of the computer to localize and lateralize the involved SCC is useful to increase the diagnostic accuracy in the cases diagnosed with vestibular lithiasis. ${ }^{19}$

I am reporting here a very unusual case of right unilateral multicanalicular vestibular lithiasis of PSC and HSC, in which a single supine head roll test elicited three different patterns of nystagmus and all with significant localizing values. The author was able to video record the diagnostic supine head roll test.

Table 1 The relative frequencies of different BPPV variants at specialty clinics reported in various studies

\begin{tabular}{|l|l|l|l|l|l|}
\hline Authors & No. of patients & PSC-BPPV & HSC-BPPV & ASC-BPPV & Multiple canals \\
\hline De la Meilleure et al, ${ }^{1} 1996$ & 287 & $78.05 \%$ & $16.38 \%$ & - & $5.57 \%$ \\
\hline Honrubia et al, ${ }^{2} 1999$ & 292 & $85.62 \%$ & $5.14 \%$ & $1.37 \%$ & $7.87 \%$ \\
\hline Macias et al, ${ }^{3} 2000$ & 259 & $93.02 \%$ & $1.94 \%$ & - & $5.04 \%$ \\
\hline Korres et al, ${ }^{4} 2002$ & 122 & $90.16 \%$ & $8.2 \%$ & $1.64 \%$ & - \\
\hline Sakaida et al, ${ }^{5} 2003$ & 50 & $56 \%$ & $38 \%$ & & $6 \%$ \\
\hline Imai et al, ${ }^{6} 2005$ & 108 & $64.82 \%$ & $33.33 \%$ & - & $1.85 \%$ \\
\hline Nakayama and Epley, ${ }^{7} 2005$ & 833 & $66.39 \%$ & $10.08 \%$ & $2.28 \%$ & $21.25 \%$ \\
\hline Cakir et al, ${ }^{8} 2006$ & 169 & $85.21 \%$ & $11.83 \%$ & $1.18 \%$ & $1.78 \%$ \\
\hline Moon et al, ${ }^{9} 2006$ & 1,692 & $60.9 \%$ & $31.9 \%$ & $2.2 \%$ & $5.0 \%$ \\
\hline Jackson et al, ${ }^{10} 2007$ & 260 & $66.9 \%$ & $11.9 \%$ & $21.2 \%$ & - \\
\hline Chung et al, ${ }^{11} 2009$ & 589 & $61.8 \%$ & $35.3 \%$ & $2.9 \%$ & - \\
\hline
\end{tabular}

Abbreviations: ASC-BPPV, anterior semicircular canal benign paroxysmal positional vertigo; HSC-BPPV, horizontal semicircular canal benign paroxysmal positional vertigo; PSC-BPPV, posterior semicircular canal benign paroxysmal positional vertigo. 


\section{Case Description}

\section{History}

A 70-year-old male presented with 1 day history of vertigo on lying supine, getting up from supine, and on assuming either of the lateral recumbent positions. There was no history of staggering, diplopia, dysarthria, difficulty in swallowing, hiccups, drooping of upper eyelids, or facial or limb weakness. There was also no history of hypertension, diabetes, coronary artery disease, hypothyroidism, jaundice, craniocervical trauma, cervical radiculopathies, cervical canal stenosis, rheumatoid arthritis, Paget's disease, ankylosing spondylitis, low back dysfunction, spinal cord injuries, or cerebrovascular disease.

\section{Examination}

The general physical examination and vitals of the patient were normal. The screening examination of the cervical spine did not reveal any limitation of movement. The examination of the back region did not reveal kyphoscoliosis. The examination of the lumbosacral spine, including straight-leg raising (SLR) test and reverse SLR test, were normal. The neurological examination revealed normal cranial nerve examination; strength was grade 5/5 in all four limbs with normal deep tendon reflexes, and bilateral plantar reflexes were flexor. The examination of the cerebellar system revealed no spontaneous or gaze-evoked nystagmus, and there was no appendicular or axial incoordination. The otoneurological examination revealed normal vertical and horizontal saccadic and smooth pursuit eye movements. The head impulse test was bilaterally normal.

Before carrying out positional tests, the presence of spontaneous nystagmus was ruled out by using takeaway Frenzel goggles. ${ }^{24}$ The Dix-Hallpike test was carried as per the published guidelines of the American Academy of Otolaryngology, Head, and Neck Surgery Foundation. ${ }^{23}$ The patient was positioned on the examination table in long-sitting, in a manner that his right side was toward its long free edge, and the distance of his bottoms from the head end of table allowed his head to hang as he was taken to the Dix-Hallpike position. The patient's head was held with both hands and was rotated 45 degrees to his right in the yaw plane. Thereupon, he was laid in such a manner that his 45 degrees right-rotated head was extended 20 degrees on the support of the author's hands to represent the right Dix-Hallpike position. In the right Dix-Hallpike position, the patient's head was maintained for 60 seconds. As the right Dix-Hallpike position did not elicit positional nystagmus, he was positioned to the upright sitting to repeat the identical sequence of events on the left side. The left Dix-Hallpike positioning also did not elicit positional nystagmus during the 60 seconds of observation in the head hanging position. The supine roll test was performed with the patient in the long sitting on the examination table. He was positioned supine with his head landing on a four-inch-thick pillow, so it got anteflexed to approximately 30 degrees in this position. A horizontal lying-down nystagmus (LDN) beating to the patient's left was observed that lasted for 23 seconds. After waiting for 30 seconds, his head was briskly rotated first to his right, which elicited after a latency of 5 seconds, a counterclockwise torsional (from examiner's perspective) upbeating positional nystagmus lasting approximately 23 seconds. Subsequently, the patient's head was brought to the neutral supine position and then briskly rotated to his left, which after a latency of 2 seconds, elicited a geotropic horizontal positional nystagmus lasting 15 seconds. The supine head roll test repeated to the right elicited after a latency of 2 seconds, a stronger geotropic horizontal positional nystagmus. The accompanying rotational vertigo during the supine roll test was more severe during the elicitation of horizontal nystagmi. Three different elicited patterns of nystagmus during a single supine head roll test localized the disease to the right HSC and PSC ( - Video 1). Video-oculography was not utilized during the positional tests.

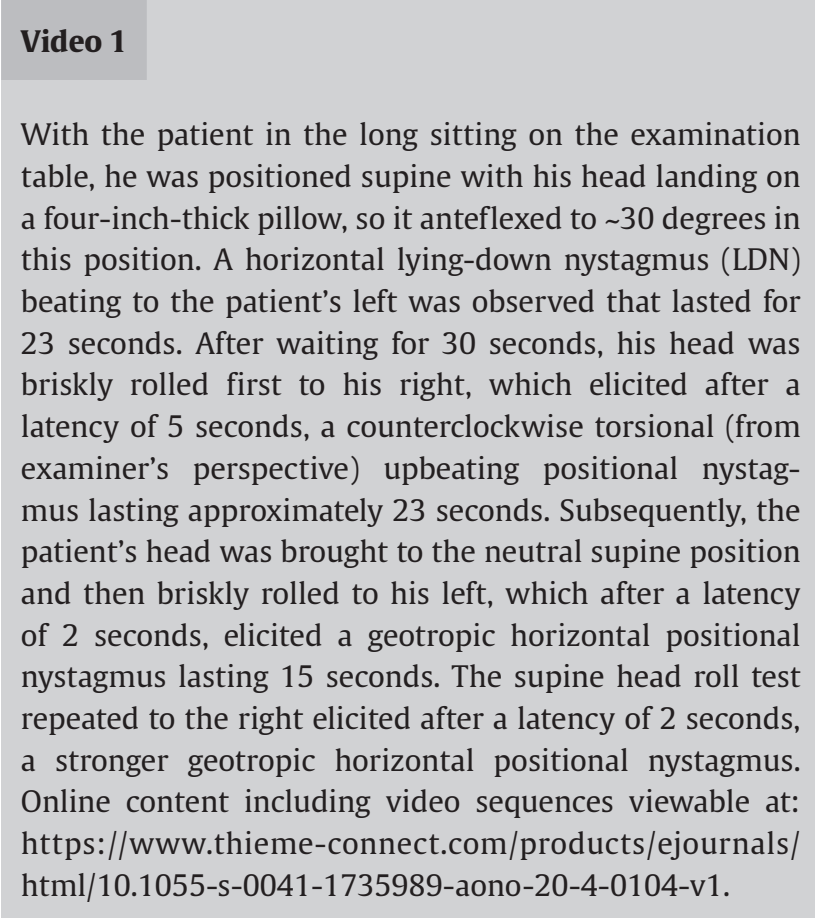

\section{Diagnosis}

The aforesaid clinical history and otoneurologic examination are consistent with the diagnosis unilateral multicanalicular be BPPV involving right-sided HSC and PSC. Video recording of the initial supine head roll test ( - Video 1), not only facilitated the identification of upbeating positional nystagmus (and hence localization to the involved PSC) but also captured a stronger horizontal geotropic nystagmus to the right (compared with the left) on repeating the supine head roll test to the right. The initial LDN to the left, captured during the video recording of the supine roll test, is also supportive of lateralization to the geotropic right horizontal semicircular canalolithiasis. The video recording of the supine head roll test was observed several times on a bigger screen of the computer to identify the stronger horizontal geotropic nystagmus, and hence the involved right HSC and PSC. 


\section{Intervention}

The patient was initially treated with Gufoni maneuver ${ }^{25-27}$ consecutively twice in one session for a total of two sessions at an interval of 1 hour, with the verifying supine roll test performed 1 hour after the second sequence of the maneuver. The Gufoni maneuver for the right geotropic HSC-BPPV was performed by making the patient sit on the edge of the examination table with both lower limbs dangling down and briskly moving the patient from sitting to the left (contralesional) lateral recumbent position and maintaining the latter position for 1 minute. Thereafter, the patient's head was rotated approximately 45 degrees downwards in the yaw plane and maintained for 2 minutes, after which he was taken to the upright sitting position. During the verifying supine roll test performed an hour after each treatment session with Gufoni maneuver, neither the geotropic positional nystagmus nor vertigo disappeared; Gufoni maneuver was deemed ineffective and abandoned thereof. Consequently, the right horizontal semicircular canalolithiasis was treated with barbecue roll maneuver (-Video 2). ${ }^{28}$ The patient was first positioned to the right lateral recumbent, for approximately 30 seconds. Thereupon, he was rolled consecutively toward his uninvolved left side, maintaining 30 seconds each in the supine, left lateral, prone, and finally right lateral recumbent positions. After completing one barbecue roll, the patient was positioned upright sitting with lower limbs dangling down the long axis of the examination table. Barbecue roll maneuver was performed six times consecutively (until two successive maneuvers were free of horizontal geotropic nystagmus during its right and left lateral recumbent positions). The patient had vomited twice during the barbecue roll maneuver and he was reassured about this. Supine head roll test was repeated an hour after the treatment session with barbecue roll maneuver. During the repeat supine head roll test, there appeared a counterclockwise torsional (from examiner's perspective) upbeating positional nystagmus after a latency of 6 seconds which lasted for 23 seconds ( - Video 3 ). LDN and geotropic horizontal positional nystagmus were conspicuously absent implying successful treatment of right horizontal semicircular canalolithiasis. For the residual right posterior semicircular canalolithiasis, the patient was treated with five consecutive modified right Epley maneuvers (EM) on the following day. The modified right EM ( $r$-MEM) was performed with the patient in the long-sitting on the examination table. A four-inch-thick pillow was placed behind his buttocks as a vantage point instead of using the end edge of the treatment table during the $r$-MEM. ${ }^{29}$ His head was rotated 45 degrees to the ipsilesional right side in the yaw axis and he was positioned supine so that his head was in 20 degrees of extension as the cervical rotation was maintained (position one). During such positioning, upbeating counterclockwise torsional nystagmus was elicited for less than a minute, with the patient experiencing concomitant vertigo for the same duration. Upon completion of 1 minute with 45 degrees of cervical rotation to the ipsilesional right side, the head was rotated 90 degrees to his left, maintaining its 20 degrees extension (position two). Positioning with 45 degrees of cervical rotation to the contralesional left side with the neck in
20 degrees of the extension, was maintained for 1 minute; patient neither complained of vertigo nor had any nystagmus during this period. Thereupon, he was instructed to assume the left lateral recumbent position and rotate his head further leftward so that his nose was positioned almost right-angled with the treatment table (position three). The latter position was maintained for 1 minute. Thereupon, he was instructed to sequentially dangle down his lower limbs along the free edge of the examination table, and while maintaining the head position, he was assisted to assume an upright sitting position completing one $r$-MEM. No vertigo complaint was made by the patient either in the nose-down left lateral recumbent position or on assuming the upright position after $r$-MEM completion. A total of five $r$-MEM were performed, with identical positional nystagmus getting elicited in position one with concurrent vertigo during the first two $r$-MEM. In the third sequence of $r$-MEM, an upbeating nystagmus was observed during position one as well three, and on assuming the upright sitting position, the patient had a severe bout of vertigo with severe anteropulsion. During the fourth and fifth $r$-MEM, neither nystagmus was observed nor vertigo was complained by the patient.

\begin{abstract}
Video 2
The patient is first positioned right lateral recumbent, for $\sim 30$ seconds. Thereupon, he is rolled consecutively toward his uninvolved left side, maintaining 30 seconds each in the supine, left lateral, prone, and finally right latera recumbent positions. After completing one barbecue roll, the patient is positioned to the upright sitting with lower limbs dangling down the long axis of the examination table. Online content including video sequences viewable at: https://www.thieme-connect.com/products/ejournals/ html/10.1055-s-0041-1735989-aono-20-4-0104-v2.
\end{abstract}

\section{Video 3}

The supine head roll test is repeated after 1 hour of the therapeutic session with barbecue roll maneuver. When the head is yawed maximally to the right, there appears a counterclockwise torsional (from examiner's perspective) upbeating positional nystagmus after a latency of 6 seconds which lasted 23 seconds. Online content including video sequences viewable at: https://www.thieme-connect.com/products/ejournals/ html/10.1055-s-0041-1735989-aono-20-4-0104-v3.

\section{Prognosis and Outcome}

A supine roll test done 20 hours after $r$-MEM did not elicit any positional nystagmus. The patient neither complained of rotational vertigo or any nonvertiginous dizziness. He was telephonically questioned weekly regarding the recurrence 
of rotational vertigo for the next 4 weeks, and it was confirmed that he remained symptom free till then.

\section{Discussion}

The otoconia within the SCCs consist of calcite crystals (in canalolithiasis as well as cupulolithiasis) composed of calcium carbonate derived as broken-off fragments from the degenerative utricular macula. Usually, only one SCC is affected, and the monocanalicular posterior semicircular canalolithiasis is by far the most frequently encountered variant. In the most common variant of unilateral multicanalicular vestibular lithiasis of PSC and HSC, possibly the otoconia enter both the canals simultaneously. By contrast, in the second most common variant of bilateral multicanalicular vestibular lithiasis of PSC and HSC, it is reasonable to think that the PSC-BPPV develops first on one side; this probably alters the habitual sleeping position of the sufferer to the unaffected lateral recumbent that sequentially leads to contralateral horizontal semicircular canalolithiasis.

The case of unilateral multicanalicular vestibular lithiasis of PSC and HSCs presented here is unusual for three reasons.

First, the clinical settings like preceding history of head trauma or underlying otologic conditions like labyrinthitis, Meniere's disease, and otitis media that predispose to the development of multicanalicular vestibular lithiasis were distinctly lacking in this case.

Second, a single supine head roll test elicited three different patterns of nystagmus (all with significant localizing values), namely horizontal LDN (to patient's left), an upbeating counterclockwise torsional (from examiner's perspective) positional nystagmus on the initial supine head roll to the patient's right, and asymmetrically strong (right more than left) geotropic horizontal positional nystagmus on the subsequent supine head roll to the patient's right as well as left. Elicitation of upbeating geotropic counterclockwise (from examiner's perspective) torsional nystagmus during the supine head roll test and not by the Dix-Hallpike test is rather unusual. Perhaps this could be due to otoconia adhering to the wall of the SCC or the individual variations in the anatomy of SCCs. Typically, when the head is yawed maximally to the right $(\boldsymbol{-}$ Fig. $\mathbf{~ 1 C}$ ) during the supine roll test, the otoconial debris within the long (posterior) arm of the right HSC falls toward the ampulla ( - Fig. 1D), eliciting a strong right beating horizontal positional nystagmus as per Ewald's second law. However, at the same time, the cohabitating otoconial debris within the ampullary arm of the right PSC is also moving ampullofugal ( - Fig. 1D) under the effect of gravity and an upbeating geotropic counterclockwise (from examiner's perspective) torsional nystagmus is anticipated as per the Ewald's third law. The elicited positional nystagmus at one point of time is the result of the net effect of force vectors generated by the otoconial movement in the concurrently involved right PSC and HSC. This explains two different patterns of the positional nystagmus elicited during yawing of head maximally to the right during initial and subsequent positionings in a single supine head roll test.

Third, Gufoni maneuver, despite its proper application in adequate numbers, failed to resolve the right horizontal
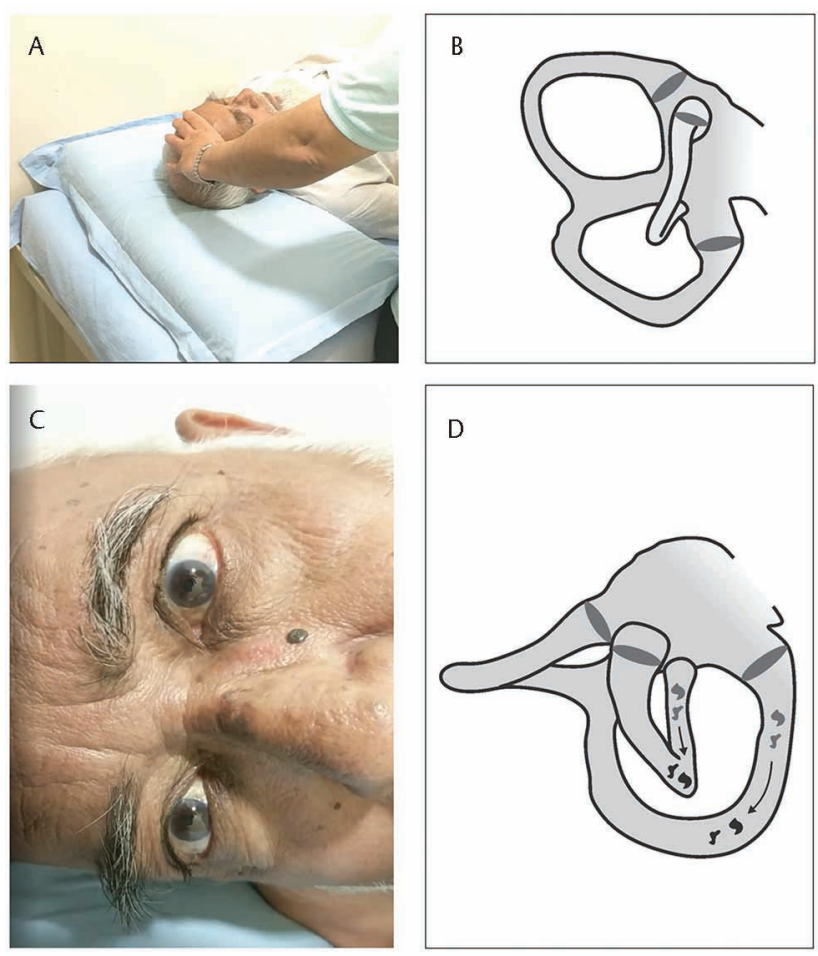

Fig. 1 (A) Supine neutral position. (B) Orientation of right-sided semicircular canals in the supine neutral position from the right of the patient. (C) Head yawed to the right during the supine roll test. (D) Orientation of right-sided semicircular canal in the supine neutral position from the right of the patient, with the head yawed maximally to the right. The otoconial debris is moved ampullopetal (straight arrow) in the horizontal and ampullofugal (curved arrow) in the posterior semicircular canals.

canalolithiasis; barbecue roll maneuver successfully treated the latter. The mastering of an alternative canalith repositioning maneuvers (CRMs) for each canal, in case of treatment failure with certain maneuvers, is mandatory for all those who are directly involved in the treatment of multicanalicular vestibular lithiasis.

The chronological sequencing of treatment with CRM, for the multicanalicular BPPV, depends on the severity of symptoms attributed to the involved SCC. It is generally agreed that the HSC is more frequently associated with autonomic symptoms like sweating, nausea, and vomiting. ${ }^{30}$ In cases of multicanalicular canalolithiasis involving PSC and HSC, a reasonable strategy would be to treat the horizontal semicircular canalolithiasis first and posterior semicircular canalolithiasis subsequently. The spatial orientation of the involved SCCs may be taken into consideration for deciding the preferential sequencing for the treatment with CRM. The PSC slopes inferiorly and has its cupular barrier at a relatively more dependent end; any otoconial debris that sequestrates in the ampullary arm of the PSC is liable to remain trapped for a long time. By contrast, the cupular barrier of the HSC is relatively higher in a location allowing free-floating debris to easily drift back to the utricle under the effect of gravity. It is, for this reason, some may prefer to treat posterior semicircular canalolithiasis first as it is believed to be the reservoir of otoconia. 


\section{Conclusion}

It is imperative to perform both positional tests, namely Dix-Hallpike maneuver, and supine head roll test, in cases suspected to have multicanalicular vestibular lithiasis. The positionings may need to be repeated several times to unveil multiple nystagmi, each with different localizing and lateralizing values. The clinician handling the case of multicanalicular vestibular lithiasis needs to realize that the pattern of nystagmus elicited during positioning in the positional test by stimulation of more than one SCC is the net effect of force vectors generated by the otoconial movement in the concurrently affected SCCs. Identifying treatment priorities with CRM for the individual SCCs is crucial. The canal that is liable to cause severe symptoms needs early clearance of the otoconial debris. In most cases of multicanalicular horizontal and posterior semicircular canalolithiasis, the former takes precedence in the order of priority. If a CRM fails to clear a SCC, an alternative maneuver may need to be executed. Clinicians involved in the care of cases with multicanalicular vestibular lithiasis should be well versed with all possible backup maneuvers for clearing each of the three SCCs.

\section{Disclaimers}

The views expressed in the submitted article are author's own and not an official position of the institution to which author is affiliated.

\section{Declaration of Interest}

The author has no disclosures to report.

\section{Conflict of Interest}

The authors certify that they have no affiliations with or involvement in any organization or entity with any financial interest (such as honoraria; educational grants; participation in speakers' bureaus; membership, employment, consultancies, stock ownership, or other equity interest; and expert testimony or patent-licensing arrangements), or nonfinancial interest (such as personal or professional relationships, affiliations, knowledge, or beliefs) in the subject matter or materials discussed in this manuscript.

\section{Acknowledgments}

To Mr. Renith Kurian who video recorded the diagnostic and therapeutic maneuvers and precisely captured the nystagmus during the entire diagnostic and treatment period and to Mr. Ashraf for drawing the - Figs. 1B and D on CorelDraw graphics suite 2019.

\section{References}

1 De la Meilleure G, Dehaene I, Depondt M, Damman W, Crevits L, Vanhooren G. Benign paroxysmal positional vertigo of the horizontal canal. J Neurol Neurosurg Psychiatry 1996;60(1):68-71

2 Honrubia V, Baloh RW, Harris MR, Jacobson KM. Paroxysmal positional vertigo syndrome. Am J Otol 1999;20(4):465-470
3 Macias JD, Lambert KM, Massingale S, Ellensohn A, Fritz JA. Variables affecting treatment in benign paroxysmal positional vertigo. Laryngoscope 2000;110(11):1921-1924

4 Korres S, Balatsouras DG, Kaberos A, Economou C, Kandiloros D, Ferekidis E. Occurrence of semicircular canal involvement in benign paroxysmal positional vertigo. Otol Neurotol 2002;23(6):926-932

5 Sakaida M, Takeuchi K, Ishinaga H, Adachi M, Majima Y. Long-term outcome of benign paroxysmal positional vertigo. Neurology 2003;60(9):1532-1534

6 Imai T, Ito M, Takeda N, et al. Natural course of the remission of vertigo in patients with benign paroxysmal positional vertigo. Neurology 2005;64(5):920-921

7 Nakayama M, Epley JM. BPPV and variants: improved treatment results with automated, nystagmus-based repositioning. Otolaryngol Head Neck Surg 2005;133(1):107-112

8 Cakir BO, Ercan I, Cakir ZA, Civelek S, Sayin I, Turgut S. What is the true incidence of horizontal semicircular canal benign paroxysmal positional vertigo? Otolaryngol Head Neck Surg 2006;134(3):451-454

9 Moon SY, Kim JS, Kim BK, et al. Clinical characteristics of benign paroxysmal positional vertigo in Korea: a multicenter study. J Korean Med Sci 2006;21(3):539-543

10 Jackson LE, Morgan B, Fletcher JC Jr, Krueger WW. Anterior canal benign paroxysmal positional vertigo: an underappreciated entity. Otol Neurotol 2007;28(2):218-222

11 Chung KW, Park KN, Ko MH, et al. Incidence of horizontal canal benign paroxysmal positional vertigo as a function of the duration of symptoms. Otol Neurotol 2009;30(2):202-205

12 Katsarkas A. Benign paroxysmal positional vertigo (BPPV): idiopathic versus post-traumatic. Acta Otolaryngol 1999; 119(7):745-749

13 Tomaz A, Ganança MM, Ganança CF, Ganança FF, Caovilla HH, Harker L. Benign paroxysmal positional vertigo: concomitant involvement of different semicircular canals. Ann Otol Rhinol Laryngol 2009;118(2):113-117

14 Korres S, Balatsouras DG, Ferekidis E. Prognosis of patients with benign paroxysmal positional vertigo treated with repositioning manoeuvres. J Laryngol Otol 2006;120(7):528-533

15 Bertholon P, Chelikh L, Tringali S, Timoshenko A, Martin C. Combined horizontal and posterior canal benign paroxysmal positional vertigo in three patients with head trauma. Ann Otol Rhinol Laryngol 2005;114(2):105-110

16 Shim DB, Song CE, Jung EJ, Ko KM, Park JW, Song MH. Benign paroxysmal positional vertigo with simultaneous involvement of multiple semicircular canals. Korean J Audiol 2014;18(3):126-130

17 Leopardi G, Chiarella G, Serafini G, et al. Paroxysmal positional vertigo: short- and long-term clinical and methodological analyses of 794 patients. Acta Otorhinolaryngol Ital 2003;23(3):155-160

18 AwST, Todd MJ, Aw GE, McGarvie LA, Halmagyi GM. Benign positional nystagmus: a study of its three-dimensional spatio-temporal characteristics. Neurology 2005;64(11):1897-1905

19 Ichijo H. Positional nystagmus of horizontal canalolithiasis. Acta Otolaryngol 2011;131(1):46-51

20 Domènech-Vadillo E, Álvarez-Morujo De Sande MG, González-Aguado R, et al. Incidence of unilateral and bilateral benign paroxysmal positional vertigo when the left and right Dix-Hallpike manoeuvres are positive: a model based on the sense of torsional nystagmus. Acta Otorhinolaryngol Ital 2020;40(2):144-151

21 Bronstein AM. Vestibular reflexes and positional manoeuvres. J Neurol Neurosurg Psychiatry 2003;74(3):289-293 
22 Ewald JR, Physiologische Untersuchungen Ueber das Endorgan de Nervus Octavus. Wiesbaden, Germany: Bergmann JF Publishers; 1892

23 Bhattacharyya N, Gubbels SP, Schwartz SR, et al. Clinical practice guideline: benign paroxysmal positional vertigo (update. Otolaryngol Head Neck Surg 2017;156(3_suppl)(suppl) :S1-S47

24 Strupp M, Fischer C, Hanß L, Bayer O. The takeaway Frenzel goggles: a Fresnel-based device. Neurology 2014;83(14):1241-1245

25 Ciniglio Appiani G, Catania G, Gagliardi M. A liberatory maneuver for the treatment of horizontal canal paroxysmal positional vertigo. Otol Neurotol 2001;22(1):66-69

26 Asprella GL, Gufoni M, Vertigine parossistica da CSL: manovre di barbecue ed altre varianti. In: Nuti D, Pagnini P, Vicini C, eds.
Atti della XIX Giornata di Nistagmografia Clinica. Milano: Formenti; 1999 321-336

27 Mandalà M, Pepponi E, Santoro GP, et al. Double-blind randomized trial on the efficacy of the Gufoni maneuver for treatment of lateral canal BPPV. Laryngoscope 2013;123(7):1782-1786

28 Tusa RJ. Vertigo. Neurol Clin 2001;19(1):23-55, v

29 Radtke A, von Brevern M, Tiel-Wilck K, Mainz-Perchalla A, Neuhauser H, Lempert T. Self-treatment of benign paroxysmal positional vertigo: Semont maneuver vs Epley procedure. Neurology 2004;63(1):150-152

30 Pagnini P, Nuti D, Vannucchi P. Benign paroxysmal vertigo of the horizontal canal. ORL J Otorhinolaryngol Relat Spec 1989;51(3):161-170 